\title{
Improving resilience management for critical infrastructures - strategies and practices across air traffic management and healthcare
}

\author{
V. Cedrini \& M. Mancini \\ ENAV S.p.A, Air Navigation Service Provider (ANSP), Rome, Italy \\ L. Rosi, G. Mandarino \& S. Giorgi \\ Italy National Health Institute (ISS), Rome, Italy
}

I. Herrera \& M. Branlat

SINTEF, Trondheim, Norway

J. Pettersson \& C.-O. Jonson

Center for Disaster Medicine and Traumatology, and Department of Clinical and Experimental Medicine, Linköping University, Linköping, Sweden

L. Save \& D. Ruscio

DeepBlue SrL, Rome, Italy

\begin{abstract}
Recent natural and man-made disasters highlight that a more resilient approach to preparing for and dealing with such events is needed. To address this challenge, the main objective of the research and innovation H2020 project DARWIN is the development of European resilience management guidelines for Critical Infrastructures (CI). Based on a systematic literature survey with a world-wide scope and prioritization of resilience concepts, the guidelines have been developed taking into account everyday operations, contingency plans, training, etc. This paper describes insights gained from the adaptation of these guidelines in the domains of Air Traffic Management (ATM) and Healthcare (HC). A collaborative and iterative process has been defined involving relevant experts and practitioners. To ensure transnational, cross-sector applicability and uptake, a Community of Crisis and Resilience Practitioners (DARWIN DCoP) has been involved. The preliminary results indicate that a big step has been taken in moving from the resilience theory to practice.
\end{abstract}

\section{INTRODUCTION}

ATM and HC have a great track record of safe operations in challenging conditions, even if disruptions or occasional crises may happen routinely. While it can certainly be improved, both domains have already implemented a number of practices and methods, especially related to being able to handle such disruptions or to learning from them. Still, recent examples from disasters are reminders of the urgent need to improve our ability to reveal, assess and manage resilience, both in everyday operations and during crises (Hollnagel et al., 2011, Adini et al, 2017).

The overall objective and main result of the Horizon 2020 EC project DARWIN is the development of European resilience management guidelines. These guidelines are called DARWIN Resilience Management Guidelines (DRMG).

The DRMG consist of suggested interventions and guiding principles to help or advise any organization in the creation, assessment or improvement of its own reference guidelines, procedures and practices.

What is really important is that DARWIN results are useful for our end users namely the Critical Infrastructures that include ATM and HC.

For this purpose, the DARWIN Resilience management guidelines are designed to address disruptions, changes and opportunities; facilitate anticipation, adaptation, flexibility; and provide a foundation for an effective crisis response (Adini et al., 2017).

An initial set of generic DRMG was produced (DARWIN D2.1, 2016) and then adapted to ATM and $\mathrm{HC}$ to make the guidelines more operational and usable in these domains.

This paper presents the approach and methodology carried out to adapt the DRMG to both domains and discusses relevant results. 


\subsection{Nature of the DARWIN guidelines}

The methodology to obtain the list of DRMG has been thoroughly defined: based on a world-wide systematic literature review carried out for the DARWIN project (DARWIN D1.1), 56 concepts, approaches and practices have been identified and evaluated (DARWIN D1.2).

The results of an evaluation following a modified Delphi process with practitioners and experts resulted in essential and important resilience concepts to be included in the resilience management guidelines. These conceptual as well as user requirements are input for the development of the DRMG (DARWIN D1.3).

The guidelines are developed as individual topics that address the conceptual requirements identified. Those topics are referred to as Concept Cards (CC). CCs propose interventions that organizations can implement (the how) to reach the resilience management capabilities captured in the conceptual requirements (the what). Through those interventions, the guidelines aim to help CI organizations in developing a critical view of their own crisis management activities (management of resources, procedures, training, etc.). The $\mathrm{CC}$ are structured in content blocks that contain information such as: purpose; interventions proposed; actors in charge; illustration; associated practices, methods and tools; etc. In addition, while they address specific aspects of resilience management, CCs are not independent and links between them are captured through various means.

DARWIN CCs, and in particular adapted CCs, could be complementary to guidelines, procedures and practices already present in the organizations of the two domains, fostering their revision, improvement or even creation of new guidelines.

Also, each CC includes a Minimum Viable Product (MVP) which is the smallest way to start using the interventions proposed in the CC. The MVP is the set of minimum set of features required to test or experiment a solution. Its purpose is to get through the "build-measure-learn" feedback cycle as quickly and efficiently as possible (Ries, 2011). The DARWIN project proposed this solution based on interactions with experts (managers and front-line operations). This approach contrasts the traditional product development of designing, performing preliminary and critical reviews, producing and testing and perfecting the product.

\subsection{Content of the DARWIN guidelines}

The DARWIN CCs are organized under the following themes:
SUPPORTING COORDINATION AND SYNCHRONIZATION OF DISTRIBUTED OPERATIONS

1. Promoting common ground in cross-organizational collaboration

2. Establishing networks for promoting inter-organizational collaboration

3. Ensuring that actors involved in resilience management have a clear understanding of their responsibilities and the responsibilities of other involved actors

Managing adaptive CaPaCity

4. Enhancing the capacity to adapt to both expected and unexpected situations

5. Establishing the capacity for adapting during crises and other events that challenge normal plans and procedures ASSESSING RESILIENCE

6. Identifying sources of resilience

7. Noticing brittleness

8. Assessing community resilience to understand and develop its capacity to manage crises

DEVELOPING AND REVISING PROCEDURES AND CHECKLISTS

9. Managing policies involving systematicallypolicy makers and operational personnel for dealing with emergencies and disruptions

INVOLVING THE PUBLIC IN RESILIENCE MANAGEMENT

10. Interacting with the public not yet affected by or involved in a crisis

\section{METHODOLOGY}

The established methodology is a systematic step by step approach strictly intertwined with the other DARWN activities. These include in particular those relevant to the development of generic guidelines, to their evaluation and to interaction with the DCoP.

The adaptation process consists of two main steps:

- Step 1: Selection of adaptable CCs, i.e. the assessment for the adaptability of the generic CCs

- Step 2: Adaptation of adaptable CCs, that is the adaptation of the generic CCs to ATM and $\mathrm{HC}$ domains, and the release of the adapted guidelines.

\subsection{Selection of adaptable CCs}

This phase has been performed by applying a methodology based on a quantitative and qualitative SWOT (Strengths, Weaknesses, Opportunities and Threats) analysis that assessed if a CC was adaptable or not. 
The SWOT analysis methodology is commonly used to develop a deep understanding of all kinds of situations in business, organizations, and for individuals, to support the decision-making process.

It is noteworthy that the SWOT analysis findings address relevant actions for Guidelines developers concerning the improvement of the generic guideline content also. In particular, during the development of DRMG, the CCs were simultaneously assessed with regard to a possible adaptation to the specific domains, possibly avoiding any gaps between development and later adaptation.

At the end of the adaptability assessment two lists are expected: the list of non-adaptable CCs including the rationale behind their non-adaptability or elements that can be improved, and the list of adaptable CCs including the rationale behind their adaptability.

The applied SWOT analysis has been defined combining the quantitative and qualitative approaches for a richer collection of data.

The quantitative SWOT analysis has been based on the definition of a set of Indicators $(I)$ that were identified starting from the fields of the $\mathrm{CC}$ used for the process of adaptation.

Seventeen indicators (Table 2) have been established, each of them formulated as a specific statement and categorized according to the four areas of the SWOT:

- the Strength/Weakness (S/W) areas include indicators concerning internal aspects of the CC (i.e. specific contents of the $\mathrm{CC}$ fields).

- the Opportunity/Threat $(\mathrm{O} / \mathrm{T})$ areas include indicators whose assessment needs to take into account a more long-term perspective and the

Table 1. Step 1 overview.

\begin{tabular}{ll}
\hline Input & $\begin{array}{l}\text { Last available version of Generic } \\
\text { CCs }\end{array}$ \\
\hline Output & List of Adaptable and non- \\
& Adaptable CCs \\
& Information concerning content \\
& for CCs adaptation (from \\
& qualitative SWOT \\
$\bullet$ & Information concerning elements \\
& of the Generic CC improvement \\
& 1-day per each interview with \\
& each ATM/HC expert \\
concerning each single CC SWOT & 3-4 days (per CC) to organize \\
relevant information and & perform additional research \\
Required & 1 day to review the results with \\
& involved expert \\
\hline
\end{tabular}

interdependency with external factors linked to the contexts of the $\mathrm{CC}$ application.

Experts' opinions on the indicators have subsequently been collected through seventeen questions formulated as follows "How much do you agree with the following statement (I_01,I_02, ...I_17)?"

The answers to each question were recorded using a 5-point Likert scale ranging from "Disagree" to "Very Strongly Agree", with "Somewhat agree" in the middle.

In order to obtain a quantitative figure for each indicator's assessment, a numeric value was assigned to each level of the scale, starting from 1 (="Disagree") to 5 (="Very Strongly Agree") and incrementing by one per level.

Thus, according to the $I$ mean value, the assessment of each I has been classified according to the criteria described below.

The qualitative SWOT analysis of each generic $\mathrm{CC}$ was carried out by collecting comments and feedback from experts during the assessment of the quantitative SWOT analysis indicators.

After the quantitative assessment of each indicator, the expert was asked to explain the rationale of the scoring, indicator by indicator, while the interviewer was taking notes.

The interview started with the narration of the illustrative case or lesson learnt that, according to the expert, better supports the discussion on the contents of the specific CC applied to the domain.

The rationales provided during the interviews have been collected and grouped into four areas of the SWOT on the basis of the mean values calculated for each indicator (ref. Table 2).

It is noteworthy that also the rationales fully contrasting the average evaluation for the specific indicator have been kept and taken into account for the sake of richness of data.

In addition to the CCs adaptability assessment, the qualitative SWOT analysis results have been considered as one of the main sources of information used to adapt the CCs' content to the specific domain. Moreover, the collected information has been enriched using sources of information available online.

Some criteria were established to evaluate the adaptability of each CC to the specific domain. They were based on two mean scores of the SWOT analysis results:

- I-02 mean score-This indicator directly refers to the applicability of the CC to the local context in which the card will be used. The applicability is the condition sine qua non the CC can be used in real ATM/HC environment.

- Total mean score of all CC indicators - This value provides a synthetic measure of the "adequacy" 


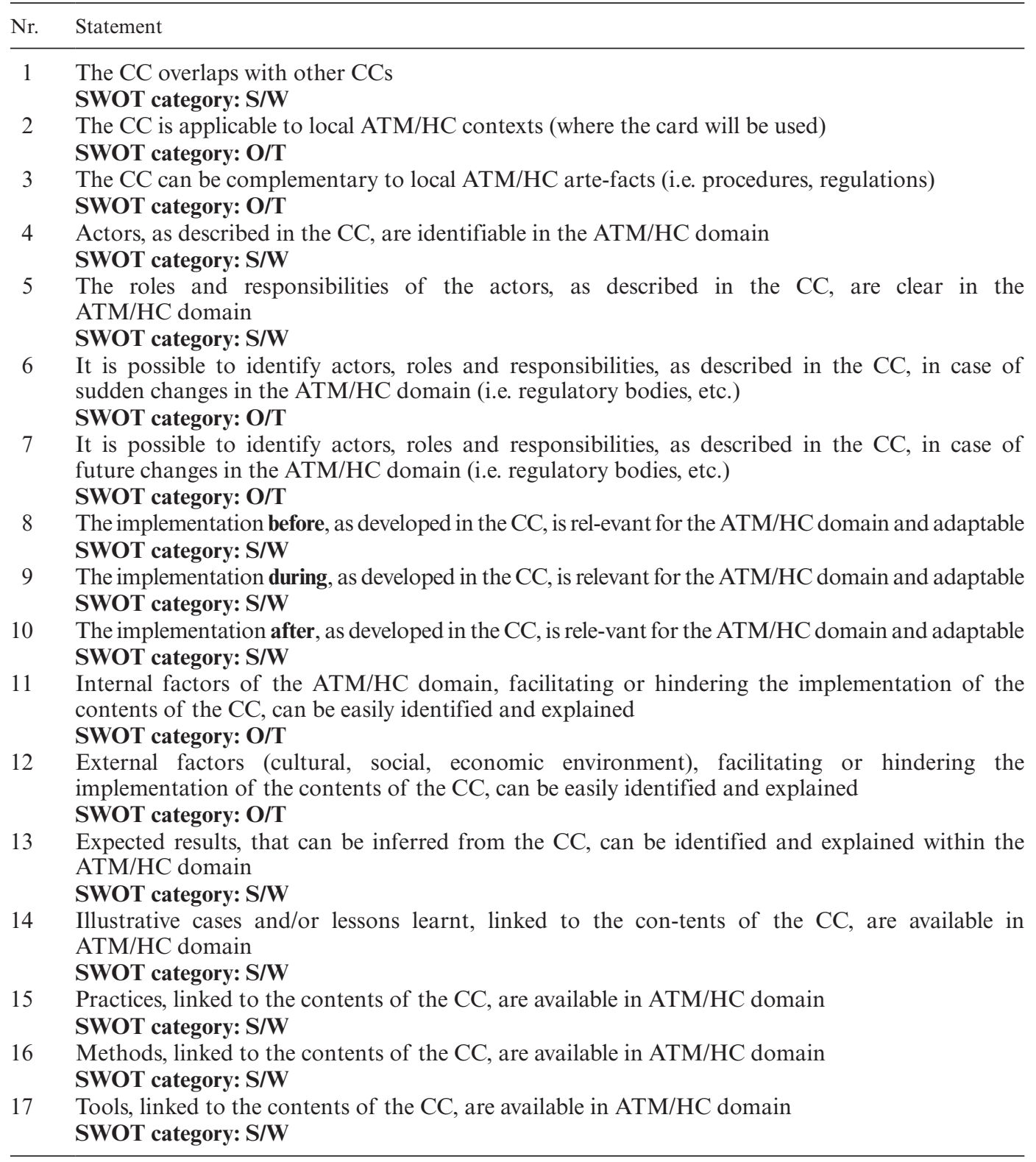

and maturity of the $\mathrm{CC}$ fields for the adaptation purposes.

The combination of these two mean scores - as explained in Figure 1 - defines the adaptability of each $\mathrm{CC}$ and specific issues to be addressed by $\mathrm{CC}$ developers.

Figure 1, Table 4 show the criteria applied to establish if a CC is adaptable or not, and the actions identified to handle the issue with guideline developers.

\subsection{Adaptation of adaptable CCs}

Once a CC has been evaluated as adaptable, the second step of the adaptation process begins. The Adapted CCs have been developed by integrating several sources:

- The findings of the qualitative SWOT analysis performed in Step 1;

- The information collected during ad-hoc interviews with domain specific experts; 
Table 3. Criteria for the classification of SWOT results.

\begin{tabular}{|c|c|}
\hline $\begin{array}{l}\text { Indicator } \\
\text { mean score }\end{array}$ & Classification of the Indicator I \\
\hline$I>3$ & $\begin{array}{l}\text { I classified as Strength or Opportunity } \\
\text { The indicator is helpful to the CC } \\
\text { adaptation to the ATM/HC domain }\end{array}$ \\
\hline$I<3$ & $\begin{array}{l}I \text { classified as Weakness or Threat } \\
\text { The indicator is "harmful" to the CC } \\
\text { adaptation to the ATM/HC domain }\end{array}$ \\
\hline$I=3$ & $\begin{array}{l}\text { Those Indicators whose mean value } \\
\text { was =3 have been classified by taking } \\
\text { into account the experts' comments } \\
\text { collected by the qualitative SWOT } \\
\text { analysis: } \\
\text { - the Indicator has been classified as } \\
\text { Strength or Opportunity if the } \\
\text { majority of the comments mainly } \\
\text { emphasized positive elements; } \\
\text { - the Indicator has been classified as } \\
\text { Weakness or Threat if the majority } \\
\text { of comments highlighted lacks and } \\
\text { missingpoints. }\end{array}$ \\
\hline
\end{tabular}

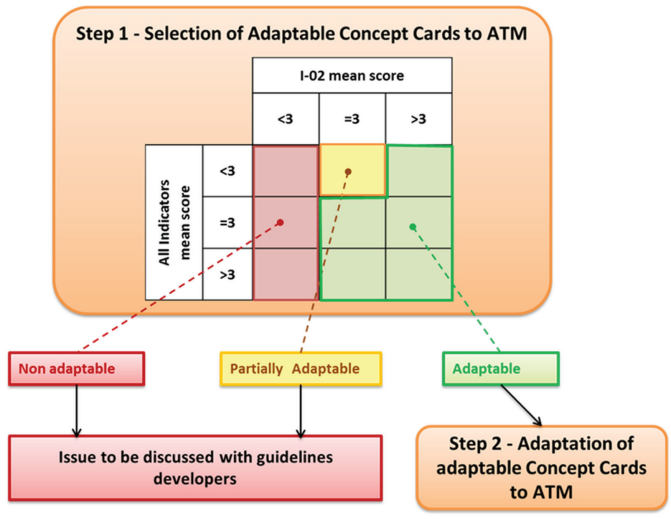

Figure 1. Adaptability Criteria.

- The information provided by the domain specific experts involved in the "initial evaluation of guidelines";

- The feedback provided by the DCoP during the workshop;

- The results collected during the implementation of Pilot exercises.

A strategic selection of participants from management as well as front line operators was performed. A template was prepared to follow a semi-structured interview with experts. The interview started with a narration of an illustrative case to better support the discussion of the context of the CC.

The topics covered concern actors involved, actions prior to, during and after the crisis. The template also includes context information, prac-
Table 4. Rationale for CC classification (example).

$\mathrm{CC}$

\begin{tabular}{ll} 
Classific. "Non-adaptable" or "Partially adaptable" \\
\hline Rationale & - the concept is valid at a general level but \\
difficult to adapt. It is not applicable in \\
the local ATM/HC domain (i.e. due to \\
type of organization and current policies \\
of the local ATM/HC systems); or \\
- the CC is not adequately developed to \\
be adapted; or \\
- so far, it has been particularly difficult \\
to find specific ATM/HC content for \\
the majority of the fields. \\
Major amendments are needed and the \\
issue has to be discussed with \\
guideline developers
\end{tabular}

$\mathrm{CC}$

Classific. "Adaptable"

Rationale $\bullet$ the $\mathrm{CC}$ is applicable in the local ATM/HC domain; or

- most of fields of the CC are adequately developed. However, in some cases some effort could be needed to make adjustments or to find specific ATM/HC content for some fields.

Action The CC can be adapted and some issues to be discussed with guidelines developers

Table 5. Step 2 overview.

\begin{tabular}{|c|c|}
\hline Input & $\begin{array}{l}\text { - Last available version of Generic CCs } \\
\text { - Output from Step 1: } \\
\text { - List of Adaptable CCs } \\
\text { - Information concerning content for } \\
\text { adaptation of CCs (from qualitative } \\
\text { SWOT) }\end{array}$ \\
\hline Output & DRMG/Adapted CCs to ATM/HC \\
\hline Effort & - 1 day (per CC) to interview one/two \\
\hline Required & $\begin{array}{l}\text { ATM/HC expert/s; } \\
\text { - } 5 \text { days (per CC) to integrate Wiki with } \\
\text { relevant information coming from SWOT, } \\
\text { expert interviews, DCoP feedback, CC } \\
\text { evaluations, feedback from pilots, } \\
\text { additional research on internet. }\end{array}$ \\
\hline
\end{tabular}

tices, methods and tools as well as other illustrative cases. The relevance of the content proposed by the $\mathrm{CC}$, in particular concerning the interventions, was discussed.

\section{RESULTS ON THE SELECTION OF ADAPTABLE CC}

The adaptability assessment of the CCs has been gradually carried out during the development process of the generic CCs. 
The SWOT analysis started as soon as the generic guidelines developers team considered the $\mathrm{CC}$ mature enough to be released and assessed for adaptation.

The information collected during the SWOT has been enriched using sources of information, suggested by the interviewee, available online and integrated in the DARWIN Wiki.

Overall review of expert feedback has provided guidance in terms of quality of the adaptable guidelines. It has been taken into consideration when updating the adaptable CC as well when elaborating new CCs.

Elements that have been mostly appreciated among experts are:

- The concepts developed by the CCs are relevant to the ATM and HC context,

- Actors in ATM and HC context are identifiable in a clear and concise manner; also roles and responsibilities are clear, being hierarchy well defined in ATM and $\mathrm{HC}$,

- The list of actions/interventions give sufficient explanation of responsibilities making it easier to adapt to the ATM, and to HC, while taking into account the broader and different fields of $\mathrm{HC}$,

- The triggering questions are useful and well grouped,

- The indications provided in the fields "implementation before/during/after" are sufficient to develop a CC adapted to the ATM and HC context,

- The level of provided information makes it easier to be integrated with local artefacts (procedures, plans),

- Useful examples, illustrative cases, practices and methods are available in the ATM and HC context.

Elements that need improvement:

- In some CCs, the information is very high level or too generic thus making it difficult to adapt,

- Some content concerning the "Triggering Questions" and "Actions" is redundant and needs to be simplified,

- No tools are provided in some of the current version of the CCs, thus, during the adaptation process, efforts should be spent, accordingly,

- Harmonization still needs to be reached among some CCs.

\section{RESULTS FROM ADAPTATION OF CC}

At first sight, ATM and $\mathrm{HC}$ seem to be very different contexts, but during meetings, the DARWIN team has discovered that they share many similarities and many common issues (i.e. criticalities of the infrastructures, impact on the public, etc.).

Notwithstanding that, the aviation domain in general is characterized by high level of standardization. The number of standards and regulations guarantee that ATM has a great track record of safe operations.

Regulatory bodies and concerned actors are well defined together with roles and responsibilities.

For example, the geographical limitation of an aerodrome makes this type of environment exposed to a relatively limited number of crisis types (e.g., aircraft accident during take-off or landing, disaster in the premises, loss of working resources, climatic event, etc.).

Although it is impossible to know when the crisis will occur, the characteristics and dynamics of crisis situations can be foreseen in advance to some degree. As a consequence, the concerned actors and the response procedures can be defined with sufficient accuracy before the crisis occurs.

On the other hand, other types of crises in ATM may be much more extended from a geographical point of view and less predictable in the way they evolve (as in the example of the Eyjafjallajökull volcano eruption in 2010).

As previously suggested, the $\mathrm{HC}$ domain exhibits common aspects with ATM that deals with criticalities and brittleness, and, in addition, they share the same scientific basis on which public health and HC tasks (i.e. care, surveillance, research, regulation and control) that are the same bases/criteria of Safety and Quality Assurance present in ATM.

What is, however, peculiar to $\mathrm{HC}$ is the individual/team resilience that $\mathrm{HC}$ workers (professional and operators) practice daily while performing their task and while coping with unexpected situations. In this case, the management of this resilient approach, i.e. systematically creating the conditions to bridge the gap between work-as-imagined and work-as-done (WAI vs. WAD), proves to be challenging.

Other relevant aspects include that this domain shows more complexity, and a variety of tasks, with many actors, ranging from surgeons to nurses, from regulatory bodies, providers, training organizations. These lead to an variety of systems, processes and outputs (protocols, documents, or records that could differ from hospital to hospital.

$\mathrm{HC}$ moves to innovation, however it should be recalled how professionals frequently support clinical decisions over standardization (a clinician sometimes stands on autonomous judgement provided for it is based on knowledge and belief).

One of the noteworthy outcomes of the adaptation process is that we discovered more uses than we expected at the beginning of the project. We found out that the guidelines are useful, they can 
be adapted and adopted in many occasions such as training, workshops and meetings.

They help to start discussions and to deal with significant topics and they can be used to:

1. Check or update current procedures and guidelines, if already existing;

2. Define new procedures and guidelines if not existing;

3. Identify possible indicators and evaluation of trends (to do possible benchmarking);

4. Prepare plans;

5. Perform risk assessment and management.

During the interviews with ATM and HC experts concerning the $\mathrm{CCs}$, some common aspects that play an important role in the resilient management of crisis emerged:

\section{- The CCs should concern all levels of ORGANIZATION}

Even if, at first sight, the DARWIN CCs may address only policy makers and management, being responsible for the modification of current procedures, it is noteworthy that all concepts address all levels of organization starting from senior management to front line operators.

- The Roles AND Responsibilities of InVOlved ACTORS CHANGE ACCORDING TO THE TYPE OF CRISIS AND THE RELATED ENVIRONMENT OF OPERATIONS

In the ATM context, according to the type of crisis several actors are involved. According to 'ICAO Annex 14. Emergency and other services', An Airport Emergency Plan shall be established to coordinate the response and participation of all existing agencies which could assist in responding to an emergency.

Examples of possible agencies $\mathrm{ON}$ and $\mathrm{OFF}$ aerodrome are provided:

ON-aerodrome: air traffic control unit, rescue and firefighting services, aerodrome administration, medical and ambulance services, aircraft operators, security services, and police;

OFF-aerodrome: fire departments, police, health authorities (including medical, ambulance, hospital and public health services), military, and harbour patrol or coast guard.

\section{- The establishment of Just Culture and Safety Culture in all Organizations}

With particular reference to the concept of "noticing brittleness", Just Culture and Safety Culture are the internal factors that could help in facilitating the identification of brittleness in each organization.

The concept of 'Just culture' is discussed in EUROCONTROL (2006) "in recent years the concept of "Just culture" has become better understood and accepted by people employed in the aviation industry. However [...] the need for a "just culture" is generally not understood by many legislators and therefore not accepted within their State judicial systems."

This issue causes "increased fear of sanctions against the reporter, particularly if partly or fully responsible for the reported occurrence."

"Furthermore, certain elements of the media may deal aggressively with apparent breaches of flight safety within certain airlines and ANSPS."

"These factors-punishing Air Traffic Controllers or pilots with fines or license suspension - may have the cumulative effect of reducing the level of incident reporting and the sharing of safety information. This hinders safety improvement and as a cascading effect resilience."

There could be concerns about possible misuse of information regarding brittleness in the organizations, since "one of the major problems with collecting and analysing information is that such information can be a very powerful tool and, like any powerful tool, if used properly it will provide great benefit. However, it can also be used improperly and if that occurs considerable harm can be caused".

In the last decade, many progresses have been made to encourage Just Culture in the European ATM context, mainly thanks to the efforts of EUROCONTROL: e.g. Air Navigation Service Providers are endorsing Just Culture policies and programmes, Task Forces have been created to promote, debate and discuss issues concerning safety and justice, meetings are organized to encourage interaction between safety and the judicial experts; special "just culture" courses for aviation experts and prosecutors have been organized, etc.

According to EUROCONTROL (2008), Safety Culture is "the way safety is perceived, valued and prioritised in an organization. It reflects the real commitment to safety at all levels in the organization. [...] It is not something you get or buy; it is something an organisation has. [...] It can therefore be positive, negative or neutral."

Since 2006, there is an active involvement of EUROCONTROL, in collaboration with FAA and CANSO, in measuring and improving Safety Culture within ANSP organizations. Safety Culture surveys are continuously planned and performed, results and recommendations are taken into account and implemented to guarantee an effective SMS and a healthy Safety Culture.

\section{- The importance of Planning, training and TESTING IN ADVANCE}

The plan should include a clear definition of the agencies involved, the responsibility and role of each agency and the coordinates of offices/people to be contacted in case of emergency. 
The training of the people allows to maintain the high level of preparedness for possible crisis events.

The test of the plan could be done in many different ways beginning with the organization of exercises, from a lower level to a higher level, with each one building on the concepts of the previous: discussion-based and operations-based exercises. The execution of the exercises allows to identify weaknesses in the plans and possibly improve them.

Discussion-based exercises are organized to discuss the plans for upcoming operations-based exercises, and to make everyone familiar with roles, procedures and responsibilities. They include: seminars, workshops, tabletop exercises, and games.

Operations-based exercises are used to validate and test plans and procedures that have been consolidated after the discussion-based exercises. They allow to better clarify roles and responsibilities of involved actors, identify gaps and limitations of the plan, and improve everyone's performance. They include drills, functional and full-scale exercises.

\section{- The IMPORTANCE OF LeSSON LEARNED DISSEMINATION}

The importance of the dissemination of the relevant information after the crisis events is fundamental in order to improve the resilience of the organization during crisis. In the ATM context and for this particular purpose, EUROCONTROL encourages the lesson learnt distribution and exchange of best practices though the website Skybrary. As well, the magazine Hindsight contains lot of useful case studies and provides the Air Traffic Controllers (ATCo) with a means to share their experiences concerning ATM-related safety occurrences. The objective is to "broaden ATCOs understanding of the problems that may be encountered, learn more about possible solutions and be better prepared in the face of similar occurrences."

Moreover, the presence of the "triggering questions" was particularly appreciated even if it may be difficult to use them during time-critical types of crisis as a checklist to be read step-by-step and to identify someone that checks their completion. On the other hand, it is important that all the actors involved in the management of the crisis are fully aware of the topics addressed.

For crises developing over a longer time (e.g. Icelandic volcano eruption or Ebola outbreak) it is possible to organize workshops and meetings to reflect with other colleagues on the possible sources of brittleness and use the triggering questions to support the reflection. The same approach can be used during a drill or a simulation by a facilitator to guide the simulation and stimulate participants to notice brittleness.

\section{DISCUSSION AND CONCLUSIONS}

The work performed so far confirms the intended readership as policy makers, front line operators, resilience engineering managers, crisis managers, critical infrastructures managers, methodologists, community of practice in ATM and HC. Stakeholders such as managers and policy makers can use this work as source of inspiration when adapting resilience guidelines to their domains.

In particular, applying the DARWIN resilience concepts, triggering questions, methods and tools, they will be able to:

- Apply the proposed interventions provided in the CCs to survey current practices, strategies, procedures and guidelines;

- Start to reflect on "what went well" and not only "what went wrong" when learning from events;

- Assess the effectiveness of roles and responsibilities during a crisis;

- Revise and/or define common action plans through periodical coordination activities and training;

- Identify brittleness in the system and the application of procedures and response to the crisis;

- Get to know practices, methods and tools applied by others;

- Test and improve their plan of communication with public during emergencies.

The collaborative method presented in this paper illustrates an iterative approach that brings theoretical concepts close to their practical implementation. We gathered information from other domains through workshops with members of the DCoP. The SWOT facilitated translation of resilience concepts into practical interventions. The methodologies proposed to adapt the concepts are defined in detail to ensure other concepts to be included in the future. The participation of experts is essential to ensure applicability, relate to the specific domain as well as enrich the cards with existing practices and methods.

At the beginning of the work, we planned separate guidelines for ATM and $\mathrm{HC}$. The first results were cards that replicated the generic cards. This overlap in relevance and adaptation in CCs outcome in ATM and $\mathrm{HC}$ indicate the potential of the generic CCs to be applicable to other sectors. The current result combines generic fields with adaptations to HC and ATM as required. The results indicate the possibilities of similar adaptations to other domains.

A challenge is the achievement of consensus on the review process and iteration to achieve sufficient maturity. Another challenge as well as an opportunity is to merge different cultural perspectives across Europe when dealing with crises. We 
found this as a window of opportunity to learn mapping recommended practices and methods within and across domains.

Further work includes evaluation of DRMG and associated CCs in relevant operational scenarios. We consider collecting feedback from ATM, $\mathrm{HC}$ as well as other domains from the DCoP.

\section{ACKNOWLEDGEMENTS}

The research leading to these results received funding from the European Union's Horizon 2020 research and innovation programme under grant agreement No 653289. Opinions expressed in this publication reflect only the authors' views and that the Agency is not responsible for any use that may be made of the information it contains. The authors would like to thank the group of professionals - both researchers and practitioners from the DARWIN project as well to experts contributing to the work related to the adaptation and paper review including other members from CARR, ENAV, FOI, ISS, KMC and SINTEF.

\section{REFERENCES}

Adini, B., Cohen, O., Eide, A.W., Nilsson, S., Aharonson-Daniel, L., Herrera, I., (2017) Striving to be resilient: What concepts, approaches and practices should be incorporated in resilience management guidelines? Journal Technological Forecasting \& Social Change. Vol 121, pp. 39-49.
DARWIN (2015). Deliverable D1.1. Consolidation of resilience concepts and practices for crisis management. Available at: http://www.h2020darwin.eu/ project-deliverables.

DARWIN (2016). Deliverable D1.3. Practitioner and academic requirements for resilience management guidelines. Available at: http://h2020darwin.eu/ project-deliverables/.

DARWIN (2016). Deliverable D2.1. Generic Resilience Management Guidelines. Available at: http://www. h2020darwin.eu/project-deliverables.

DARWIN (2017). Deliverable D2.2. Generic Resilience Management Guidelines Adapted to Healthcare Domain. By the end of 2017, available at: http://www. h2020darwin.eu/project-deliverables.

DARWIN (2017). Deliverable D2.3. Generic Resilience Management Guidelines Adapted to Air Traffic Management Domain. By the end of 2017, available at: http://www.h2020darwin.eu/project-deliverables.

EUROCONTROL ESARR advisory material/guidance document (EAM/GUI) - EAM 2/GUI 6 "Establishment of 'just culture' principles in ATM safety data reporting and assessment".

EUROCONTROL (2008) "Safety Culture in ATM-An overview".

EUROCONTROL Hindsight Magazine [https://www. eurocontrol.int/content/hindsight].

Hollnagel, E., Pariès, J., Woods, D.D., Wreathall, J., 2011. Resilience Engineering in Practice: A Guidebook. Farnham, (2011). Resilience Engineering Perspectives volume 3. Ashgate Publishing, UK: Ashgate., UK.

ICAO Annex 14 "Emergency and other services"

Reis, E. (2011). The Lean Start-up. How today's entrepreneurs use continuous innovation to create radically successful businesses. 